\title{
Improved Protein Transfer Efficiency and Signal Intensity in BlotMan Using Pulse Width Modulation
}

\author{
Thuc Nhi Truongvo', Feixue Zheng', Stanley Chien ${ }^{2,3}$, Hiroki Yokota ${ }^{1,2^{*}}$ \\ ${ }^{1}$ Department of Biomedical Engineering, Indiana University Purdue University Indianapolis, Indianapolis, IN, \\ USA \\ ${ }^{2}$ YC Bioelectric LLC, Indianapolis, IN, USA \\ ${ }^{3}$ Department of Electrical and Computer Engineering, Indiana University Purdue University Indianapolis, \\ Indianapolis, IN, USA \\ Email: "hyokota@iupui.edu
}

Received 22 March 2016; accepted 23 April 2016; published 26 April 2016

Copyright (C) 2016 by authors and Scientific Research Publishing Inc.

This work is licensed under the Creative Commons Attribution International License (CC BY). http://creativecommons.org/licenses/by/4.0/

(c) (i) Open Access

\begin{abstract}
BlotMan is a protein blotting device that allows generating multiple membranes from a single polyacrylamide gel. To transfer all proteins uniformly with the same efficiency regardless of protein size, BlotMan employs pulse-width-modulated (PWM) voltage that applies a higher average voltage to a larger protein species. BlotMan can be controlled not only by its custom-made interface but also by a smart phone via Bluetooth technology. In this study, we examined effects of PWM signals $(50 \%, 60 \%$, and $80 \%$ duty cycle) on transfer efficiency and signal intensity in comparison to a constant voltage signal $(100 \%$ duty cycle). The result revealed that in response to the same average voltage of $150 \mathrm{~V}$, a lower duty cycle with a higher maximum voltage increased transfer efficiency as well as sharpness of transferred proteins. We validated BlotMan's capability using a chondrosarcoma cell line (SW1353 cells) and a breast cancer cell line (MDA-MB231 cells) in response to anti-tumor chemical agents. BlotMan successfully generated 5 membranes from a single gel and detected 5 protein species such as c-Src, eukaryotic translation initiation factor 2 alpha (eIF2), phosphorylated eIF2, lamin B, and actin. Collectively, we demonstrated herein that BlotMan reduces an amount of protein samples by generating multiple membranes from a single gel and improving signal intensity with PWM voltage signals.
\end{abstract}

\section{Keywords}

Pulse Width Modulation (PWM), Western Blotting, Protein Transfer

\footnotetext{
${ }^{*}$ Corresponding author.
}

How to cite this paper: Truongvo, T.N., Zheng, F.X., Chien, S. and Yokota, H. (2016) Improved Protein Transfer Efficiency and Signal Intensity in BlotMan Using Pulse Width Modulation. J. Biomedical Science and Engineering, 9, 269-279. 


\section{Introduction}

Western blotting is the most widely used technique to determine the relative amount of proteins [1] [2]. Although it has been well established for over 30 years, the procedure remains highly labor-and-resource intensive. New products for quick reactions, better imaging, and capillary electrophoresis for fully automation have been released [3] [4]. Few technologies, however, are being proposed to improve an existing transfer procedure. Although capillary electrophoresis presents a new procedure, it may induce potential problems such as capillary clogging, air bubble formation, sensitivity to temperature fluctuations, inconsistency in polymer quality, and background noise in antibody reactions [5].

To elevate efficiency of Western blotting, we have previously developed BlotMan that enables to generate multiple blotting membranes from a single polyacrylamide gel [6] [7]. In a conventional Western blotting procedure, a single gel is used to make a single membrane. If three protein species need to be analyzed, for instance, three gels are used. BlotMan is able to generate three membranes from a single gel, and thus it contributes to significant reduction in labor and resources.

One potential drawback for generating multiple membranes from a single gel is signal intensity. Since the protein of interest is equally distributed to multiple membranes, an amount of protein each membrane receives becomes $1 / \mathrm{N}$ in which $\mathrm{N}$ is the number of membranes. In this study, we addressed a question: Does a PWM voltage signal ( $<100 \%$ duty cycle) increase signal intensity and sharpen protein bands compared to a constant voltage signal (100\% duty cycle)? PWM has two major parameters: frequency and duty cycle, and we herein focused on potential effects of duty cycle on signal intensity.

In the newly designed BlotMan, we re-designed a user interface and made it possible to control the device using a cell phone via Bluetooth technology [8]-[10]. Using a breast cancer cell line (MDA-MB231 cells), we validated usefulness of BlotMan in protein expression analysis in cancer biology.

\section{Materials and Methods}

BlotMan generates multiple membranes with uniform transfer efficiency for all proteins regardless of protein size from a single. It applies a voltage gradient in which the large protein receives high voltage signals via pulse width modulation. In this study, the voltage from the power supplier was set to constant at $28 \mathrm{~V}$, and the actual voltage applied to proteins in the gel was varied up to $24 \mathrm{~V}$ with a variable duty cycle. For instance, an average voltage of $12 \mathrm{~V}$ was achieved with a maximum voltage of $24 \mathrm{~V}$ and $50 \%$ duty cycle.

\subsection{Hardware and Software}

BlotMan consists of three main components: a protein transfer unit, a controller unit, and a smart phone (Figure 1(a)). The protein transfer unit consists of a multi-anode board and a cathode board (Figure 1(b)). The controller unit is a microcontroller based system. This unit receives user commands through keypad/LCD user interface or Bluetooth from an Android smart phone, and generates PWM signals of different duty cycles to multiple anodes. The Bluetooth communication between the smart phone and the controller is supported by the BlotMan application on the smart phone (Figure 1(c)). This application sends experiment commands to the controller unit, monitors experiment status, collect experiment information from the controller, and store experiment data on the smart phone. This application supports three operations: gel electrophoresis, multi-blot, and power provision (Figure 1(d)). Unique to BlotMan is pulse width modulation (PWM) signals (Figure 1(e)), in which the signal is in a form of an on-and-off rectangle wave.

\subsection{Bluetooth Communication Protocol}

The Bluetooth communication protocol was used between an Android smart phone and a controller unit (Figure 2). The protocol included bi-directional communication paths. One is from a smart phone to a controller unit, while the other from the controller unit to the smart phone. The message frame includes the attributes such as "source ID, destination ID, message type, sub type, sequence ID, message length, payload, and checksum” (Figure 1(f)). The "source ID” and "destination ID” are unique MAC addresses, and they are used to identify the sender and receiver. The "message ID” and "sub type” are used to identify message categories. The operation command and data are saved in "payload”, while "sequence ID”, “message length”, and "checksum” are for message encoding/decoding and error checking. 


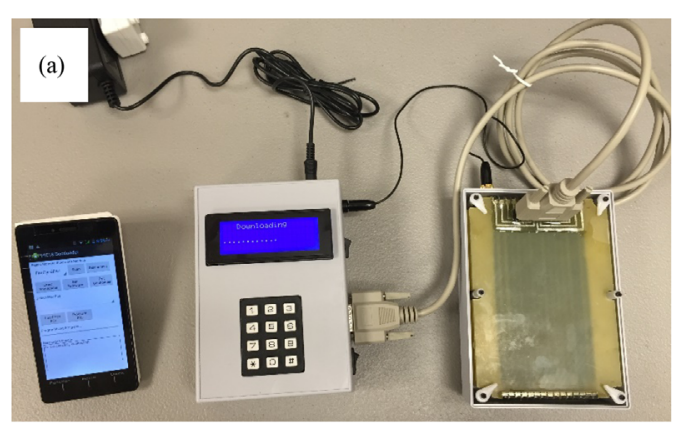

(c)

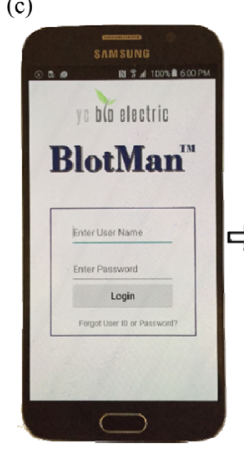

(e)

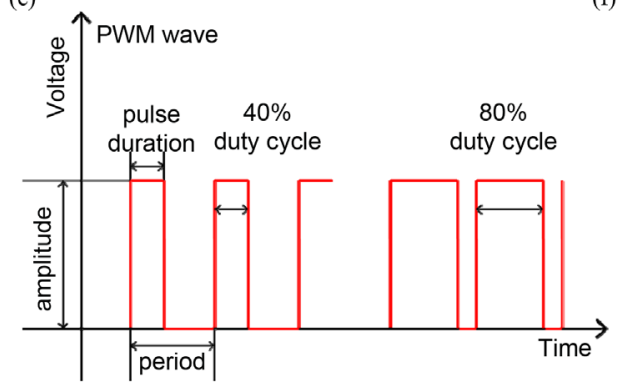

(f)

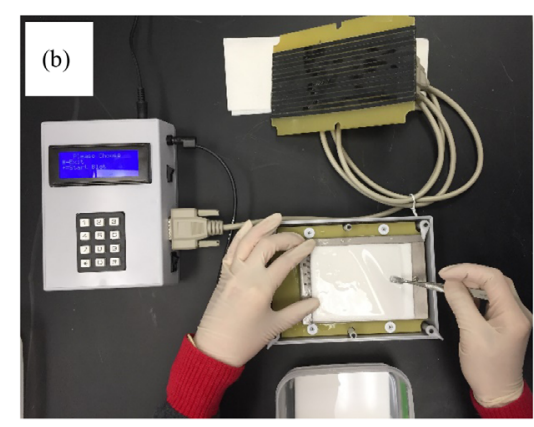

(d)

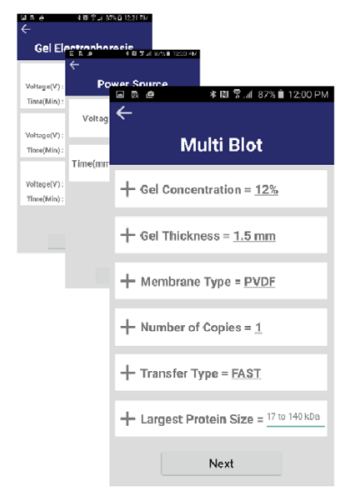

Next

Bluetooth Communication Protocal

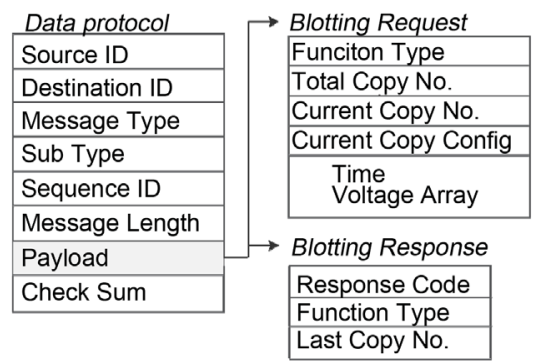

Figure 1. BlotMan system. (a) BlotMan together with a cell phone and a protein transfer unit; (b) Procedure for placing a gel on the protein transfer unit; (c) Cell phone monitor screen for operating BlotMan; (d) Interface on a monitor screen; (e) \& (f) PWM voltage profiles.

\subsection{Vertical Gel Electrophoresis}

Prior to blotting, proteins were size-fractionated by vertical gel electrophoresis. In this study, we employed two sets of protein markers in the range of $17 \mathrm{kDa}$ to $140 \mathrm{kDa}$, Spectra ${ }^{\mathrm{TM}}$ multicolor (or rainbow) makers (Thermo Scientific, Waltham, MA, USA) and Blue protein standard markers (BioLabs, Ipswich, MA, USA). Using 10\% or $12 \%$ polyacrylamide gel with 1.0 or $1.5 \mathrm{~mm}$ thickness, vertical electrophoresis was performed with a BioRad electrophoresis system (Mini-Protein ${ }^{\circledR} 3$ cell) and in a SDS-running buffer (BioRad, $25 \mathrm{mM}$ Tris, $192 \mathrm{mM}$ glycine, $0.1 \% \mathrm{SDS}$, and $\mathrm{pH}$ 8.3). We initially applied $100 \mathrm{~V}$ for $10 \mathrm{~min}$ followed by an average voltage at $150 \mathrm{~V}$ for 45 min with duty cycles of 50\%, 60\%, 80\% and 100\%. The migration distance, $d$ in mm, was modeled:

$$
d=a_{1}+a_{2} m+a_{3} / \sqrt{m}
$$

where $m=$ protein size $(\mathrm{kDa})$, and $a_{1}, a_{2}$, and $a_{3}$ were three parameters. Equation (1) was employed to predict protein positions in the gel as well as in the blotting membrane.

\subsection{Evaluation of Protein Transfer Time}

Through systematic experiments using proteins in the range of 17 - $140 \mathrm{kDa}$, we first determined blotting time to 


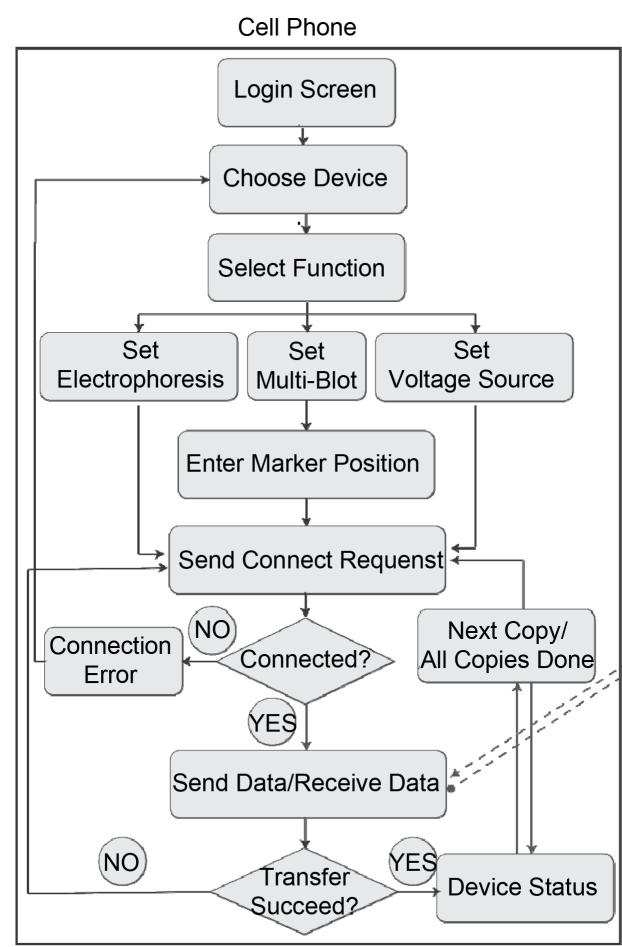

(a)

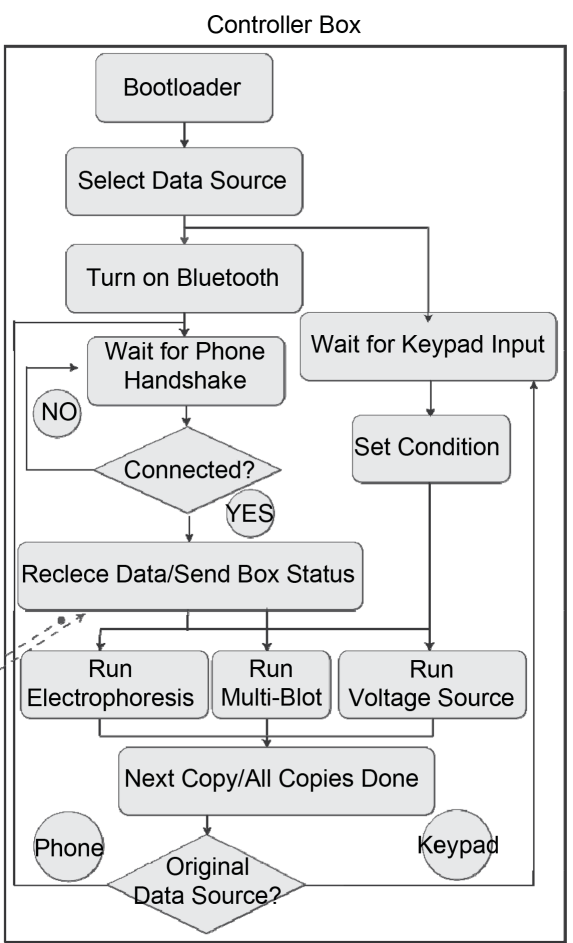

(b)

Figure 2. Flow chart for communication between a cell phone and BlotMan (controller box).

a polyvinyllidene diflouride membrane (IPVH00010, Millipore) under constant voltage signals. The transfer was conducted with a semi-dry transfer system (Transfer-Blot ${ }^{\circledR}$ cell; BioRad) at a constant voltage of 5, 7, 10, 12, and $15 \mathrm{~V}$ for 10, 20, 30, 45, 60, 120, and $180 \mathrm{~min}$ in a transfer buffer (25 mM Tris, $192 \mathrm{mM}$ glycine, pH 8.3 with $20 \%$ methanol). We approximated signal intensity of protein bands using a Gaussian distribution function and determined mean, $\mu$, and standard deviation, $\delta$, of transfer time for protein species at each of transfer voltages.

A mean transfer time, $T(\mathrm{~min})$, was then modeled using $m_{p}=$ protein size $(\mathrm{kDa})$, and $V_{t}=$ transfer voltage $(\mathrm{V})$ :

$$
\begin{gathered}
T_{m}=k_{1} m_{p}^{\alpha}+k_{2} V_{t}^{\beta}+k_{3} \\
V_{t}=\left(\frac{-k_{1}}{k_{2}} m_{p}^{\alpha}+\frac{T_{m}-k_{3}}{k_{2}}\right)^{1 / \beta}
\end{gathered}
$$

where $k_{1}, k_{2}, k_{3}$, and were parameters and determined from experimental data. The five parameters were determined using a best-fit regression approach and the mean-square error between the experimental and predicted values was minimized. Of note, $50 \%$ of proteins were expected to be transferred in $T_{m}$ regardless of protein size.

\subsection{Application to Breast Cancer Research}

A human chondrosarcoma cell line (SW1353) and a human breast cancer cell line (MDA-MB-231) were obtained from American Type Culture Collection and employed in this study [11] [12]. Cells were cultured in the presence and absence of chemical compounds and lysed in a radio-immunoprecipitation assay (RIPA) buffer that consisted of protease inhibitors (Santa Cruz Biotechnology, Santa Cruz, CA, USA) and phosphatase inhibitors (Calbiochem, Billerica, MA, USA). Isolated proteins were size-fractionated in a polyacrylamide gel by vertical electrophoresis and blotted to multiple polyvinylidene diflouride membranes (Millipore, Billerica, MA, USA) by BlotMan. After incubation for $1 \mathrm{~h}$ with primary antibodies, membranes were incubated for 45 min with secondary antibodies conjugated with horseradish peroxidase (Cell Signaling, Danvers, MA, USA). We used antibodies against c-Src, eIF2 $\alpha$, p-eIF2, lamin B (Cell Signaling), and ${ }^{\circ} \beta$-actin (Sigma). Protein signals were quantified using LAS-3000. Of note, c-Src is a proto-oncogene tyrosine kinase that is linked to cancer growth 
[13], while the phosphorylation of eIF2 is known to reduce proliferation of SW1353 and MDA-MB-231 cells

[11] [14]. Lamin B is a component in a nuclear envelope, and -actin was employed as a control.

\section{Results}

\subsection{Protein Migration in Vertical Gel Electrophoresis}

Using $10 \%$ and $12 \%$ polyacrylamide gels, size-dependent migration of proteins in a vertical gel was characterized. We defined the distance between the largest and smallest proteins (i.e., $17 \mathrm{kDa}$ and $140 \mathrm{kDa}$ ) to be a unit distance, and plotted the best fit curve for positions of other proteins between 0 and 1 (Figure 3(a)). The predicted migration distance indicates that the relative separation was greater for large proteins (>80 kDa) in 10\% gel, while a better separation was achieved for small proteins ( $<40 \mathrm{kDa})$ in $12 \%$ gel. This observation is consistent with a regular practice in which a high \% gel is employed for analyzing small proteins. The migration distance, characterized here, became a basis for determining position dependent voltage signals for protein blotting.
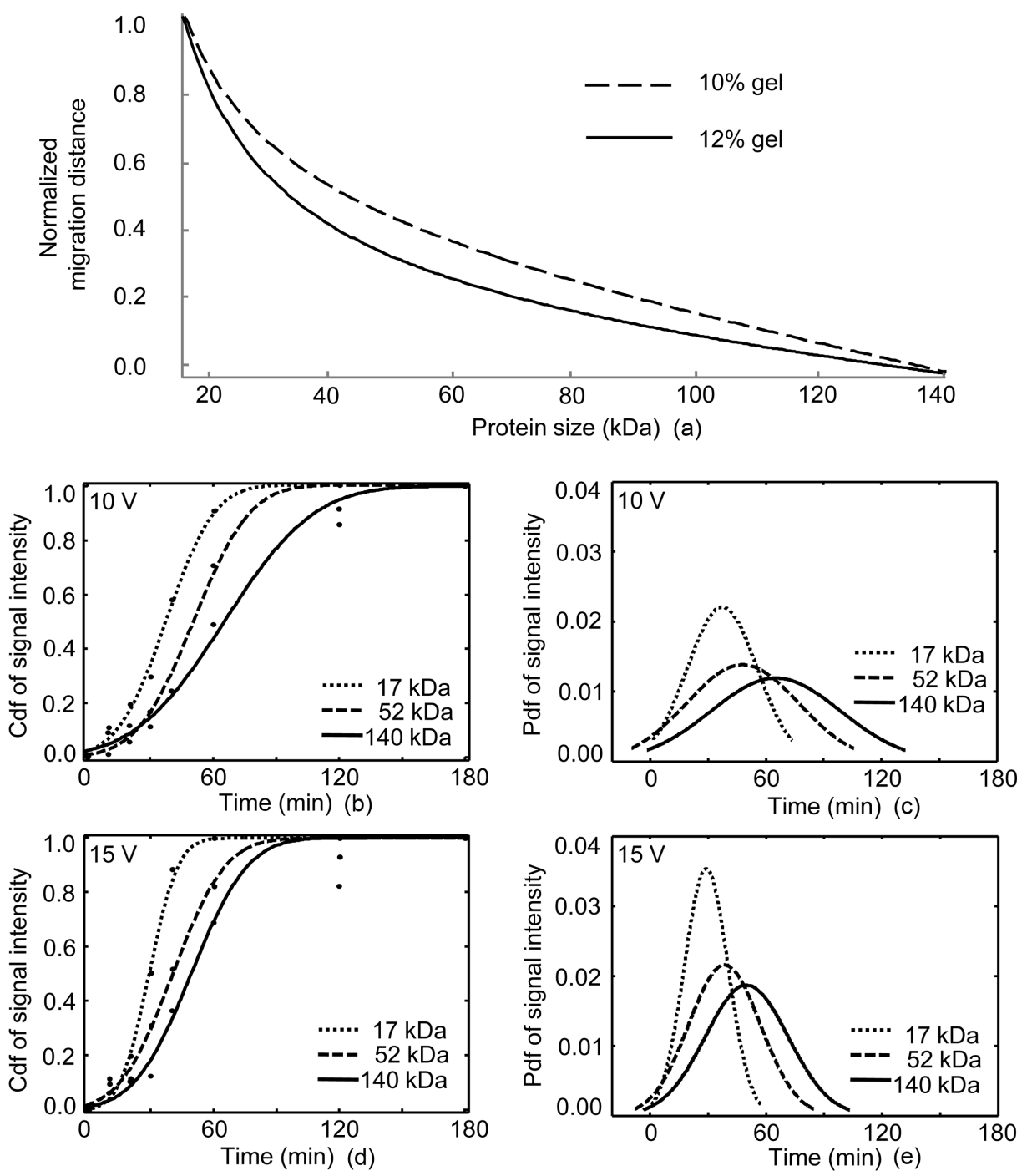

Figure 3. Size fractionation in a polyacrylamide gel and blotting to a polyvinylidene difluoride membrane. (a) Normalized protein positions via vertical gel electrophoresis in 10 and $12 \%$ gels. (b) \& (c) Blotting efficiency at $10 \mathrm{~V}$ for three protein species $(17,52$, and $140 \mathrm{kDa})$. The cumulative distribution function (cdf) and probability distribution function (pdf) were approximated by Gaussian distribution. (d) \& (e) Blotting efficiency at $15 \mathrm{~V}$ for three protein species $(17,52$, and $140 \mathrm{kDa})$. 


\subsection{Determination of Voltage Signals}

To uniformly transfer all proteins regardless of their size, BlotMan applies PWM signals with a high duty cycle to large proteins, and vice versa. To determine size dependent voltage signals, we first evaluated transfer efficiency of varying proteins ( 17 to $140 \mathrm{kDa}$ ) under constant voltages at $5-15 \mathrm{~V}$. We then determined a gradient of voltages that achieved equal transfer efficiency for all proteins. Transfer efficiency ( $0 \%$ to $100 \%)$ was plotted in a form of the cumulative distribution function (cdf), and the probability distribution function (pdf) was predicted as a time derivative of cdf (Figures 3(b)-(e)). To ease a differentiation process, we approximated cdf using a Gaussian distribution.

For a given transfer voltage for each of the selected protein species in the range of 17 to $140 \mathrm{kDa}$, a mean value of the pdf predicted a mean transfer time. Namely, $50 \%$ of proteins are expected to be transferred during the predicted mean transfer time and all proteins are completed to be transferred in twice of the mean transfer time. As expected, the mean transfer time is longer for larger proteins, while the higher voltage shortens the transfer time (Figure 4).

\subsection{Effective PWM Voltage Signals for Generating Multiple Membranes}

Based on the relationship among transfer voltage, protein size, and mean transfer time (Figure 4), we selected protein size dependent voltages that achieved the same transfer efficiency for all proteins. This size dependent voltage was generated using PWM signals, in which the larger proteins received higher duty cycle. Using BlotMan with PWM signals, we generated multiple membranes from a single gel. For instance, the case for generating 2, 3, and 5 membranes from a single gel is presented (Figures 5(a)-(c)). Among 5 membranes, we evaluated
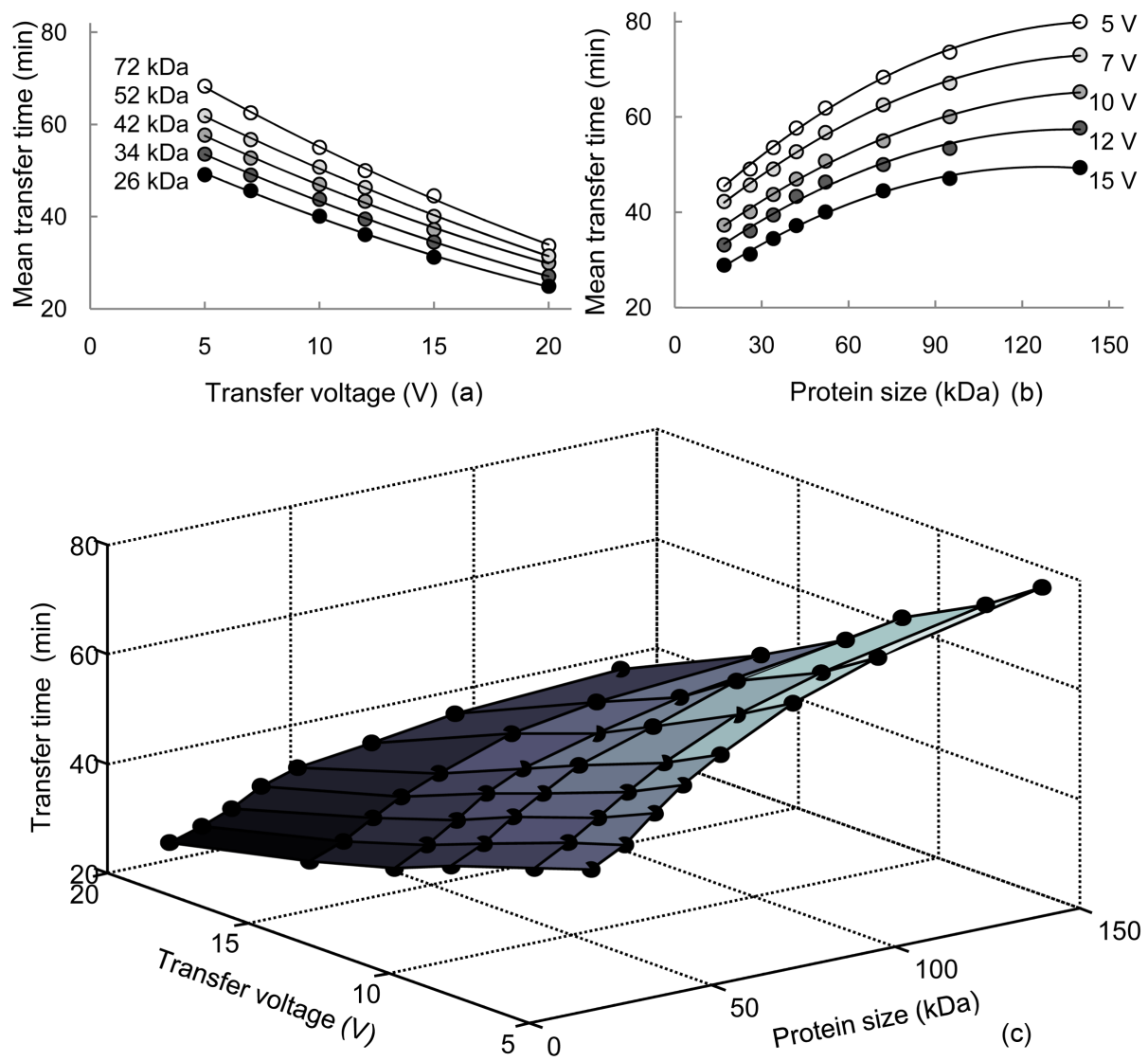

Figure 4. Relationship among mean transfer time, protein size, and transfer voltage. (a) Mean transfer time as a function of transfer voltage (5, 7, 10, 12, and $15 \mathrm{~V}$ ); (b) Mean transfer time as a function of protein size (26, 34, 42, 52, and $72 \mathrm{kDa}$ ); (c) Mean transfer time as a function of transfer voltage and protein size. 


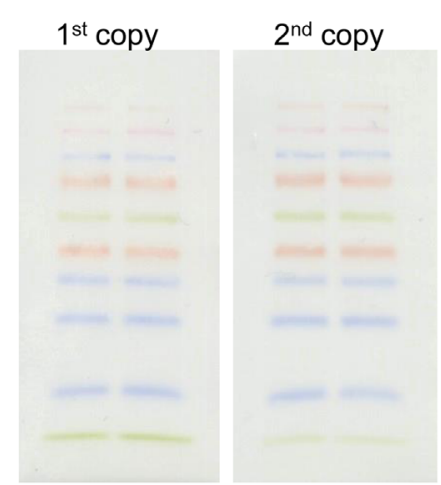

(a)

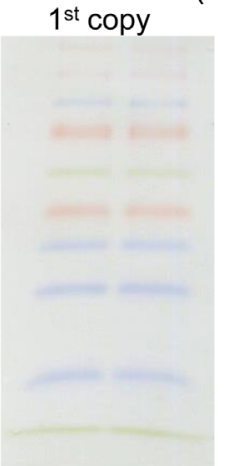

$2^{\text {nd }}$ copy

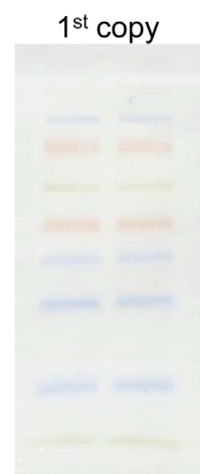

$3^{\text {rd }}$ copy

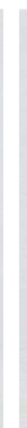

$2^{\text {nd }}$ copy

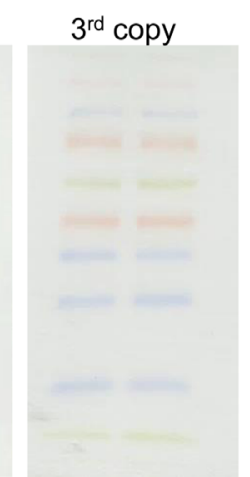

(b)

$4^{\text {th }}$ copy

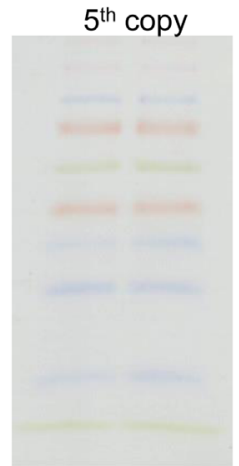

(c)

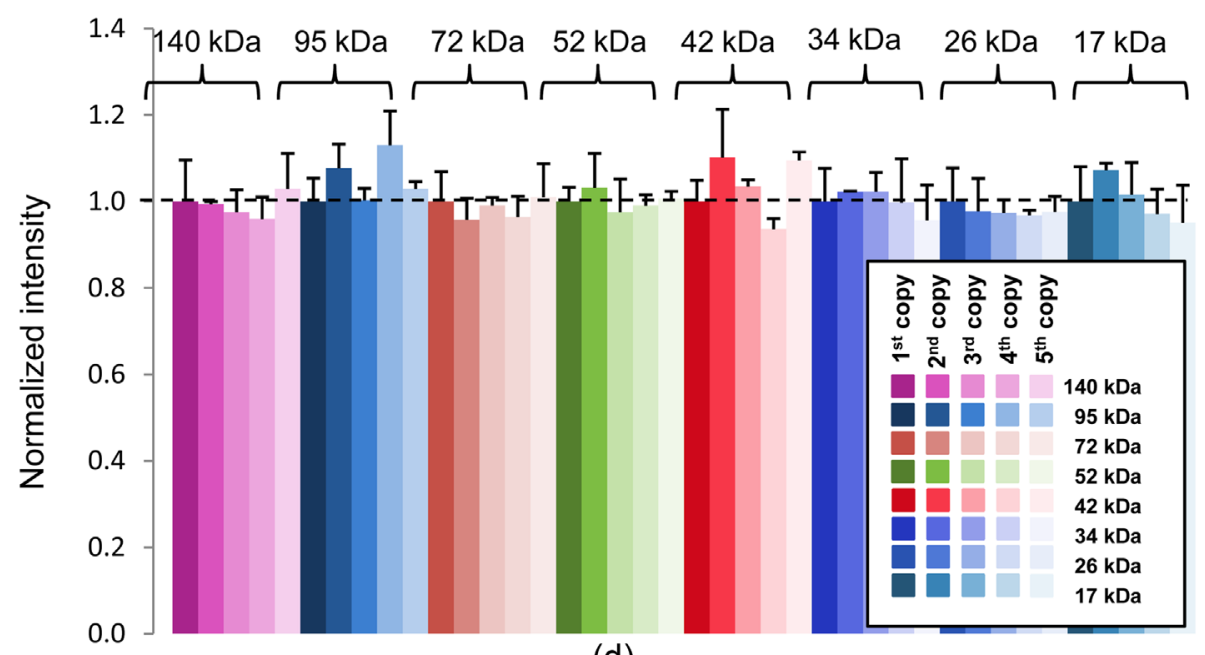

(d)

Figure 5. Generation of multiple membranes from a single polyacrylamide gel. (a) Generation of two membranes from a single gel. (b) Generation of three membranes from a single gel; (c) Generation of five membranes from a single gel; (d) Variations of blotting intensities among five membranes from a single gel.

signal intensities in the blotted membranes for 8 protein species in the range of 17 to $140 \mathrm{kDa}$ (Figure 5(d)). The result shows that signal intensities in the blotted membranes were largely uniform with an average variation of $5.2 \%$

\subsection{Improved Signal Intensity by PWM}

Signal intensity of blotted proteins is affected not only by a blotting process with BlotMan but also by electrophoresis in the vertical gel. We applied PWM signals to vertical electrophoresis as well as protein blotting, and 
evaluated the effects of PWM in the vertical gel on signal intensity of blotted proteins. When the duty cycle of the power supply for the vertical gel was reduced from $100 \%$ to $80 \%, 60 \%$ and $50 \%$ without changing the average voltage at $150 \mathrm{~V}$, the migration distance monotonously increased (Figure 6(a) \& Figure 6(b)). The signal intensity (ratio of the peak height to the width at the 50\% peak height) was approximately $39 \%$ larger for the duty cycles at $50 \%, 60 \%$ and $80 \%$ than that at $100 \%$ (Figure 6(c)).

\subsection{Application to Drug Development for Cancer Treatment}

In order to evaluate the capability of BlotMan, we employed two cancer cell lines (SW1353 chondrosarcoma, and MDA-MB-231 breast cancer) and generate five membranes from each of the two cell lines. In the first experiment with SW1353 chondrosarcoma, we prepared two samples including a control sample and a sample treated with an agent A (copies 1 to 5). All membranes were used to detect -actin (Figure 7(a)). In the second experiment with MDA-MB-231 breast cancer), each of the five membranes were used to detect different proteins such as c-Src, p-eIF2, eIF2, Lamin B, and -actin in the presence and absence of Agents b and c (Figure 7(b)). Both Agents b and c up regulated the level of p-eIF2, while Agent c (not Agent b) slightly down regulated the level of c-Src after $4 \mathrm{~h}$. The levels of eIF2, lamin B, and -actin were not significantly altered.

\section{Discussion}

This study using BlotMan demonstrates that PWM voltage signals in a vertical gel increase protein mobility as
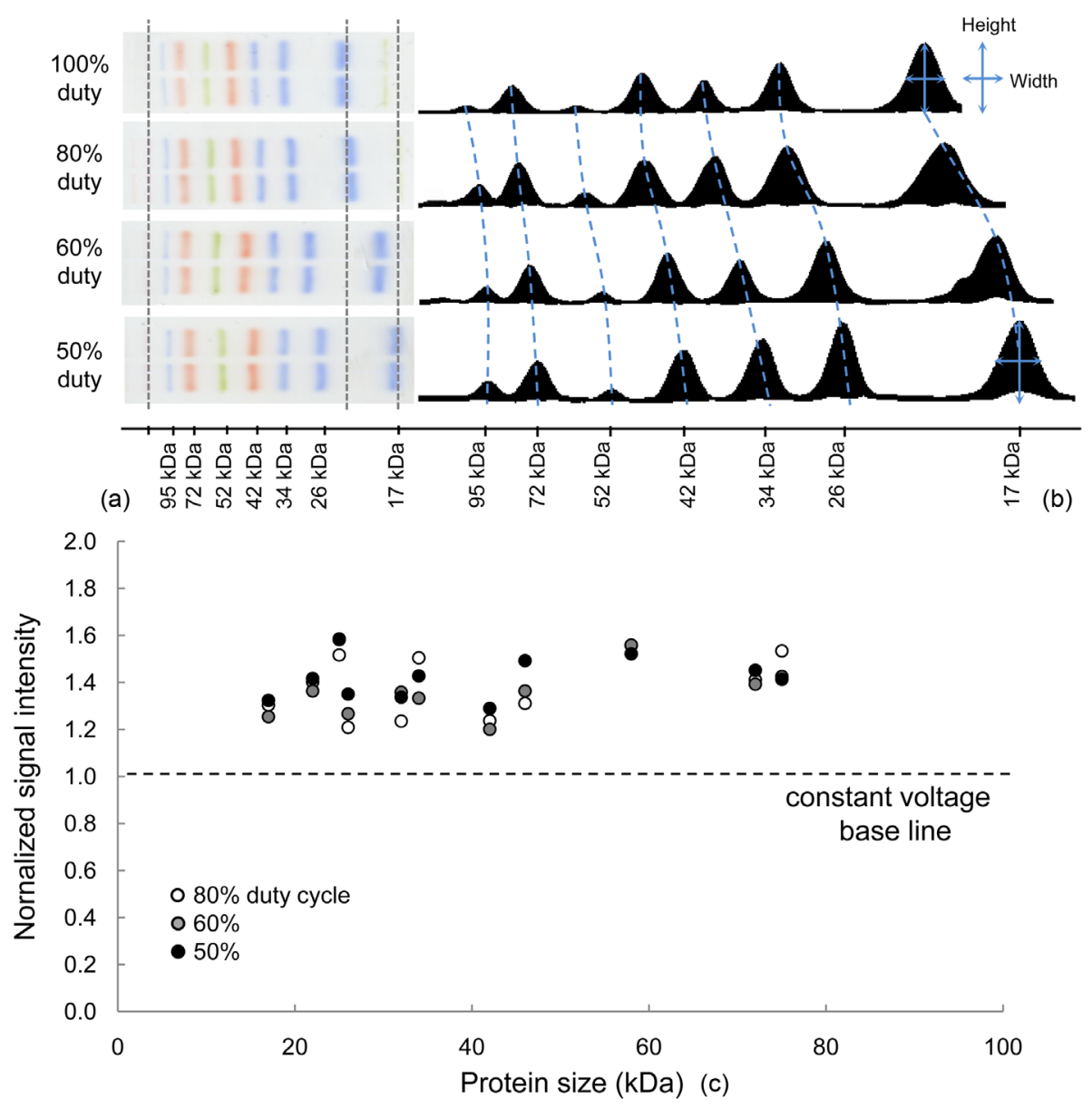

Figure 6. Effects of PWM on migration distance and signal intensity. (a) Migration distance of 7 protein species in a vertical gel in response to $50 \%, 60 \%, 80 \%$, and $100 \%$ duty cycles; (b) Normalized signal intensity of varying protein species. The unit intensity level corresponds to the case with $100 \%$ duty cycle. 


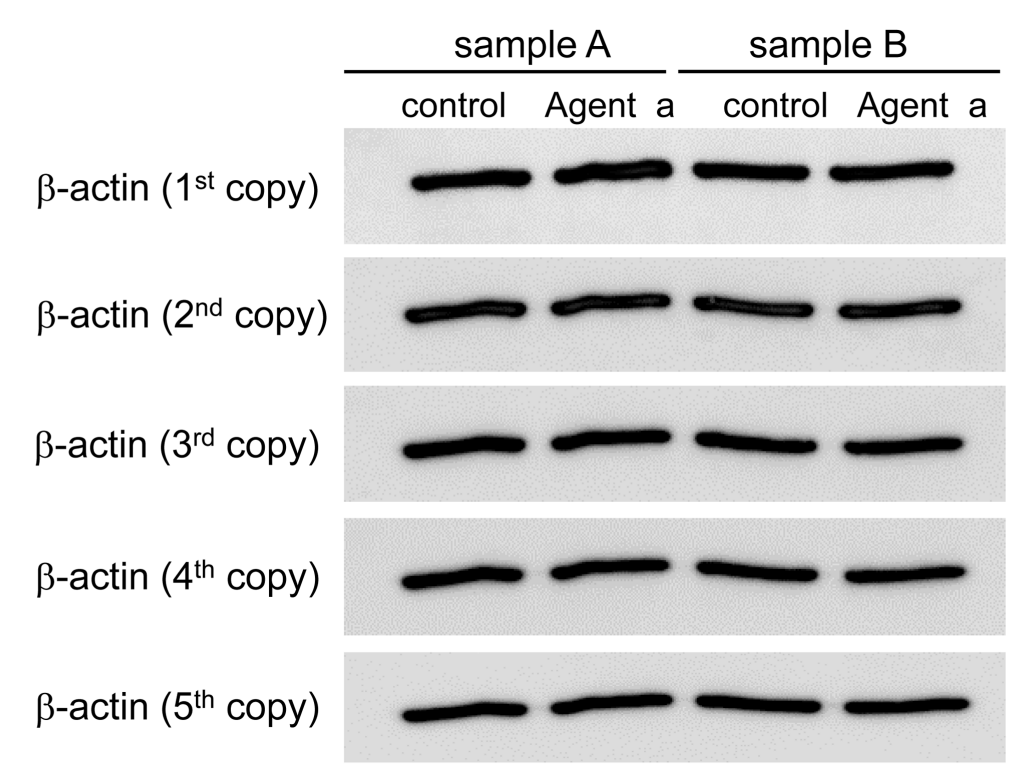

(a)

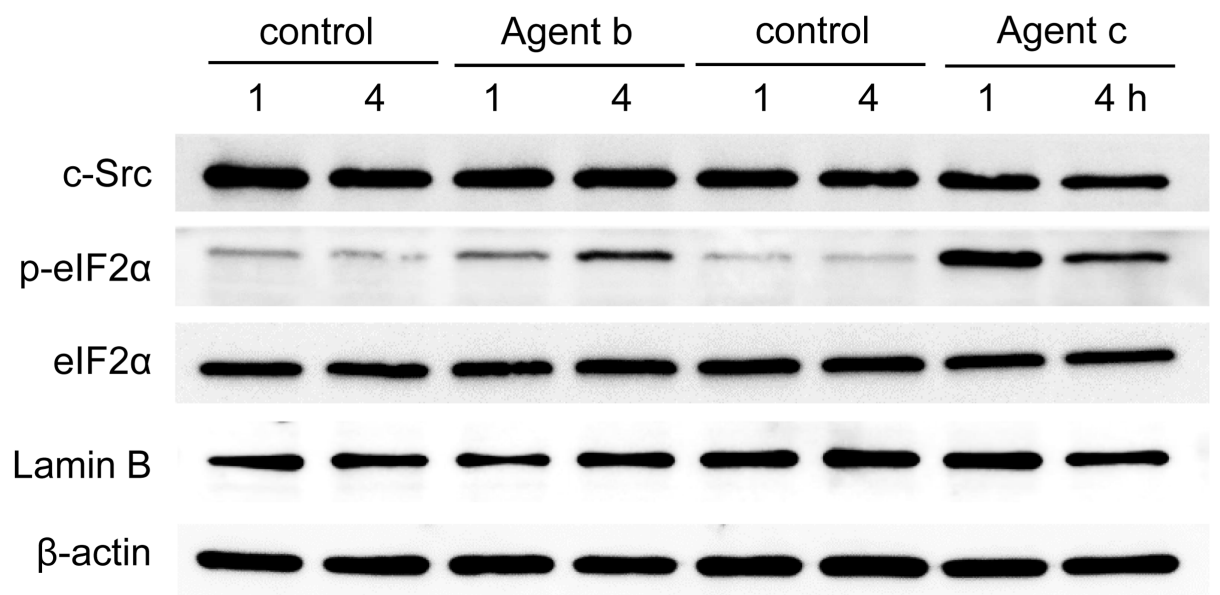

(b)

Figure 7. Detection of protein levels in 5 membranes from a single gel using two cancer cell lines. (a) Detection of -actin using protein samples isolated from SW1353 chondrosarcoma cells. (b) Detection of c-Src, p-eIF2, eIF2, lamin B, and -actin using protein samples isolated from MDA-MB-231 breast cancer cells.

well as signal intensity. In a standard gel in this study (12\% with $1.5 \mathrm{~mm}$ thickness) with an average voltage of $150 \mathrm{~V}$, the protein migration distance was increased significantly by $22 \%, 16 \%$ and $4 \%$ for the duty cycle of $50 \%, 60 \%$, and $80 \%$, respectively (Figure 6(a) and Figure 6(b)). Compared to the case with constant voltage ( $100 \%$ duty cycle), the normalized signal intensity (sharpness of bands) was improved by $43 \% \pm 9 \%$ (50\% duty cycle), $38 \% \pm 12 \%$ (60\% duty cycle), and 39\% $\pm 13 \%$ (80\% duty cycle) among the protein markers (Figure 6(c)). In a transfer of rainbow colored protein markers, 8 proteins in the range of 17 to $140 \mathrm{kDa}$ were transferred to 5 membranes from a single gel in a uniform fashion. The overall variation in 8 rainbow colored proteins among 5 copies was 5.2\%, while the variation in antibody reactions for b-actin was 5.1\% (Figure 5).

Two examples using chondrosarcoma and breast cancer cell lines showed that BlotMan is capable of analyzing 5 different protein species from a single gel without sacrificing signal intensity and quality (Figure 7). Three agents (Agents a-c) are drug candidates for attenuating tumor growth, and they are shown to regulate a signaling pathway mediated by eIF2 and its phosphorylated isoform, p-eIF2. The result clearly shows that without changing the protein level of -actin, which serves as a control, Agents b and c elevated the level of p-eIF2 after 1 and 4 
$\mathrm{h}$ treatment.

Understanding the mechanism of PWM's action on improving protein migration as well as sharpening signal intensity needs further investigation [15] [16]. We observed that PWM signals uniformly elevated migration distance of all proteins. This elevation might be linked to the effects of pulsed-field electrophoresis, which was originally developed to achieve size-dependent migration of large DNA molecules [17]. Although signal intensity was improved for all proteins, a degree of improvement differed among proteins. The observed difference may result from protein size or protein conformation.

Bluetooth technology has been rapidly utilized in the biomedical field because of the growing number of smart phone users [18] [19]. As a safe and effective method to exchange data for wireless personal area networks in short distance, it excellently matches BlotMan's user environment. Compared to the alternative WIFI (IEEE802.11x) technology, advantages of Bluetooth technology include its low power consumption, easy usage, low costs, and reduction in hardware requirements. To our knowledge, BlotMan is the first blotting device that employs Bluetooth technology for controlling protein transfers. We designed a user-friendly BlotMan application tool to facilitate the experimental procedure (i.e., gel electrophoresis, multi-blot, and power determination). For a multi-blot procedure, for example, the first step is to collect experiment information from the user and calculate PWM voltage signals. The second step is to generate the command and send it to the controller through Bluetooth. When the controller receives the request message, it sends back a response and initiates blotting. The third step is to monitor the blotting status and guide the user to conduct the blotting process.

In order to improve PWM voltage signals and further enhance BlotMan's capability, we may need to evaluate the effect of PWM frequency (in Hz). In this study, we employed $1 \mathrm{~Hz}$ but other frequencies may provide better transfer efficiency and signal intensity. In summary, we presented the improved multi-blot Western device, BlotMan, which has a novel interface with a smart phone. Compared to conventional Western blot devices, BlotMan is capable of shortening a transfer time, sharpening protein bands, and generating multiple membranes from a single gel. The device has a user-friendly interface, including a smart-phone linked controller that is activated with Bluetooth technology. Sharpening protein bands is particularly important to elevate a signal-tonoise ratio and maker are proteins at a low concentration detectable after antibody reactions. Two examples with chondrosarcoma and breast cancer cells validated uniform and clear protein signals in 5 membranes. We expect that BlotMan will contribute to improving blotting quality and saving samples, time, and costs in protein analysis.

\section{Acknowledgements}

The authors thank Billy Chien for technical assistance. This study was support in part by NIHR41GM106494 and in part by Indiana Economic Development Corporation.

\section{References}

[1] Towbin, H., Staehelin, T. and Gordon, J. (1979) Electrophoretic Transfer of Proteins from Polyacrylamide Gels to Nitrocellulose Sheets: Procedure and Some Applications. Proceedings of the National Academy of Sciences of the United States of America, 76, 4350-4354. http://dx.doi.org/10.1073/pnas.76.9.4350

[2] Burnette, W.N. (1981) Western Blotting: Electrophoretic Transfer of Proteins from Sodium Dodecyl Sulfate-Polyacrylamide Gels to Unmodified Nitrocellulose and Radiographic Detection with Antibody and Radioiodinated Protein. Analytical Biochemistry, 112, 195-203. http://dx.doi.org/10.1016/0003-2697(81)90281-5

[3] Mathews, S.T., Plaisance, E.P. and Kim, T. (2009) Imaging Systems for Westerns: Chemiluminescence vs. Infrared Detection. Methods in Molecular Biology, 536, 499-513. http://dx.doi.org/10.1007/978-1-59745-542-8_51

[4] Zhu, Z., Lu, J.J. and Liu, S. (2012) Protein Separation by Capillary Gel Electrophoresis: A Review. Analytica Chimica Acta, 709, 21-31. http://dx.doi.org/10.1016/j.aca.2011.10.022

[5] Guttman, A. (1995) On the Separation Mechanism of Capillary Sodium Dodecyl Sulfate Gel Electrophoresis of Protein. Electrophoresis, 16, 611-616. http://dx.doi.org/10.1002/elps.1150160198

[6] Hagyousif, A.M., Chong, V.J., Yokota, H. and Chien, S. (2010) Development of a Novel Protein Multi-Blotting Device. Journal of Biomedical Science and Engineering, 3, 1125-1132. http://dx.doi.org/10.4236/jbise.2010.312146

[7] Blair, M., Wanis, M., Swarnkar, G., Yokota, H. and Chien, S. (2012) Simultaneous Multi-Protein Detection Device for Western Blot Analysis. Journal of Biomedical Science and Engineering, 5, 542-547.

http://dx.doi.org/10.4236/jbise.2012.59067 
[8] Marani, R. and Perri, A.G. (2012) Design of Advanced Electronic Biomedical Systems. International Journal of Advances in Engineering and Technology, 4, 15-25.

[9] Kadlec, M.W., You, D., JoLiao, J.C. and Wong, P.K. (2013) A Cell Phone-Based Microphotometric System for Rapid Antimicrobial Susceptibility Testing. Journal of Laboratory Automation, 19, 258-266. http://dx.doi.org/10.1177/2211068213491095

[10] Erikson, D., O’Dell, D., Jiang, L., Oncescu, V., Gumus, A., Lee, S., Mancuso, M. and Mehta, S. (2014) Smartphone Technology Can Be Transformative to the Deployment of Lab-on-Chip Diagnostics. Lab on a Chip, 14, 3159-3164. http://dx.doi.org/10.1039/C4LC00142G

[11] Xu, W., Wan, Q., Na, S., Yokota, H., Yan, J. and Hamamura, K. (2015) Suppressed Invasive and Migratory Behaviors of SW1353 chondrosarcoma cells through the regulation of Src, Rac1 GTPase, and MMP13. Cellular Signaling, 27, 2332-2342. http://dx.doi.org/10.1016/j.cellsig.2015.08.014

[12] Nikkhah, M., Strobl, J.S., Schmelz, E.M., Roberts, P.C., Zhou, H., et al. (2011) MCF10A and MDA-MB-231 Human Breast Basal Epithelial Cell Co-Culture in Silicon Micro-Arrays. Biomaterials, 32, 7625-7632. http://dx.doi.org/10.1016/j.biomaterials.2011.06.041

[13] Biscardi, J.S., Belsches, A.P. and Parsons, S.J. (1998) Characterization of Human Epidermal Growth Factor Receptor and c-Src Interactions in Human Breast Tumor Cells. Molecular Carcinogenesis, 21, 261-272. http://dx.doi.org/10.1002/(SICI)1098-2744(199804)21:4<261::AID-MC5>3.0.CO;2-N

[14] Hamamura, K., Minami, K., Tanjung, N., Wan, Q., Koizumi, M., Matsuura, N., Na, S. and Yokota, H. (2014) Attenuation of Malignant Phenotypes of Breast Cancer Cells through eIF2 $\alpha$-Mediated Downregulation of Rac1 Signaling. International Journal of Oncology, 44, 1980-1988.

[15] Westerhuis, W.H., Sturgis, J.N. and Niederman, R.A. (2000) Reevaluation of the Electrophoretic Migration Behavior of Soluble Globular Proteins in the Native and Detergent-Denatured States in Polyacrylamide Gels. Analytical Biochemistry, 284, 143-152. http://dx.doi.org/10.1006/abio.2000.4684

[16] Slater, G.W. (2009) DNA Gel Electrophoresis: The Reputation Model. Electrophoresis Supplies, 1, S181-S187. http://dx.doi.org/10.1002/elps.200900154

[17] Lalande, M., Noolandi, J., Turmel, C., Rousseau, J. and Slater, G.W. (1987) Pulsed-Field Electrophoresis: Application of a Computer Model to the Separation of Large DNA Molecules. Proceedings of the National Academy of Sciences of the United States of America, 84, 8011-8015. http://dx.doi.org/10.1073/pnas.84.22.8011

[18] Vashist, S.K., Schnerder, E.M. and Luong, J.H. (2014) Commercial Smartphone-Based Devices and Smart Applications for Personalized Healthcare Monitoring and Management. Diagnostics, 4, 104-128. http://dx.doi.org/10.3390/diagnostics4030104

[19] Abbas, Z. and Yoon, W. (2015) A Survey on Energy Conserving Mechanisms for the Internet of Things: Wireless Networking Aspects. Sensors, 15, 24818-24847. http://dx.doi.org/10.3390/s151024818 